\title{
On the Behavior of the Positive Solutions of the System of Two Higher-Order Rational Difference Equations
}

\author{
Qi Wang', Gengrong Zhang ${ }^{2 *}$, Linlin $\mathrm{Fu}^{2}$ \\ ${ }^{1}$ College of Science, Guangxi University of Science and Technology, Liuzhou, China \\ ${ }^{2}$ College of Mathematics and Information Science, Guangxi University, Nanning, China \\ Email: ${ }^{*}$ grzaw@gxu.edu.cn
}

Received February 21, 2013; revised March 21, 2013; accepted April 1, 2013

Copyright (C) 2013 Qi Wang et al. This is an open access article distributed under the Creative Commons Attribution License, which permits unrestricted use, distribution, and reproduction in any medium, provided the original work is properly cited.

\section{ABSTRACT}

We study the convergence of the positive solutions of the system of the following two difference equations:

$$
x_{n+1}=\frac{x_{n-2 k+1}}{A y_{n-k+1} x_{n-2 k+1}+\alpha}, y_{n+1}=\frac{y_{n-2 k+1}}{B x_{n-k+1} y_{n-2 k+1}+\beta}, n \geq 0,
$$

where $k$ is a positive integer, the parameters $A, B, \alpha, \beta$ and the initial conditions are positive real numbers. Our results generalize well known results in [1,2].

Keywords: System of Difference Equations; Convergence; Positive Solution; Behavior

\section{Introduction}

Difference equations and the system of difference equations play an important role in the analysis of mathematical models of biology, physics and engineering. The study of dynamical properties of nonlinear difference equations and the system of difference equations have been an area of intense interest in recent years (for example, see [1-11]).

In [1], Kurbanl, Ģinar and Yalçinkaya studied the behavior of the positive solutions of the following system of difference equations

$$
x_{n+1}=\frac{x_{n-1}}{y_{n} x_{n-1}+1}, y_{n+1}=\frac{y_{n-1}}{x_{n} y_{n-1}+1}, n \geq 0 .
$$

In [2], Stevo Stević investigated the system of the following difference equations

$$
x_{n+1}=\frac{a x_{n-1}}{b y_{n} x_{n-1}+c}, y_{n+1}=\frac{\alpha y_{n-1}}{\beta x_{n} y_{n-1}+\gamma}, n \geq 0 .
$$

Motivated by the above studies, in this note, we consider the system of the following difference equations

$$
x_{n+1}=\frac{x_{n-2 k+1}}{A y_{n-k+1} x_{n-2 k+1}+\alpha}, y_{n+1}=\frac{y_{n-2 k+1}}{B x_{n-k+1} y_{n-2 k+1}+\beta}, n \geq 0,
$$

\footnotetext{
"Corresponding author.
}

where $k$ is a positive integer, the parameters $A$, $B, \alpha, \beta$ and the initial conditions are positive real numbers.

System (1) is a particular case of the system of the following difference equations

$$
\begin{aligned}
& x_{n+1}=\frac{a_{1} x_{n-2 k+1}}{b_{1} y_{n-k+1} x_{n-2 k+1}+c_{1}}, \\
& y_{n+1}=\frac{a_{2} y_{n-2 k+1}}{b_{2} x_{n-k+1} y_{n-2 k+1}+c_{2}}, n \geq 0 .
\end{aligned}
$$

If $a_{1} a_{2}=0$, then system (2) is trivial. If $a_{1} a_{2} \neq 0$, system is reduced to system (1) with $A=\frac{b_{1}}{a_{1}}, \alpha=\frac{c_{1}}{a_{1}}, B=\frac{b_{2}}{a_{2}}$ and $\beta=\frac{c_{2}}{a_{2}}$. Hence from now on, we will consider system (1).

On the other hand, system (1) is a natural generalizetion of the equation

$$
x_{n+1}=\frac{x_{n-2 k+1}}{A x_{n-k+1} y_{n-2 k+1}+\alpha}, n \geq 0,
$$

where $k$ is a positive integer, the parameters $A, \alpha$ are positive real numbers. Hence the results which we obtained can also apply to (3).

The essential problem we consider in this paper is the behavior of the positive solutions of system (1). We es- 
tablish the convergence of the positive solutions of system (1). To some extent, this generalizes the results obtained in $[1,2]$. It is interesting that a modification of our method enables us to investigate the form of the positive solutions of system (1).

\section{Main Results}

For convenience, set $r=\alpha \beta$,

$$
l_{n}=\left\{\begin{array}{ll}
n, & \text { when } r=1, \\
\frac{1-r^{n}}{1-r}, & \text { when } r \neq 1 .
\end{array} \text { for } n \geq 0\right.
$$

Let $\left\{\left(x_{n}, y_{n}\right)\right\}_{n=-2 k+1}^{\infty}$ be a positive solution of (1). Set

$$
x_{n}=\frac{1}{s_{n}}, y_{n}=\frac{1}{t_{n}} \text { for } n \geq-2 k+1,
$$

then (1) translates into

$$
s_{n+1}=\alpha s_{n-2 k+1}+\frac{A}{t_{n-k+1}}, t_{n+1}=\beta t_{n-2 k+1}+\frac{B}{s_{n-k+1}}, n \geq 0 .
$$

Set

$$
\begin{gathered}
p_{1, j, n}=\alpha+\frac{A}{r^{n} t_{-k+j} s_{-2 k+j}+l_{n}(\beta A+B)}, \\
q_{1, j, n}=\beta+\frac{B}{r^{n} s_{-k+j} t_{-2 k+j}+l_{n}(\alpha B+A)}, \\
p_{2, j, n}=\alpha+\frac{A}{r^{n}\left(\beta s_{-k+j} t_{-2 k+j}+B\right)+l_{n}(\beta A+B)}, \\
q_{2, j, n}=\beta+\frac{B}{r^{n}\left(\alpha t_{-k+j} s_{-2 k+j}+A\right)+l_{n}(\alpha B+A)},
\end{gathered}
$$

for $n \geq 0, j=1,2,3, \cdots, k$.

Lemma 2.1 For (5), we have

$$
\begin{aligned}
& s_{2 k n+j}=s_{-2 k+j} \prod_{i=0}^{n} p_{1, j, i}, t_{2 k n+j}=t_{-2 k+j} \prod_{i=0}^{n} q_{1, j, i}, \\
& S_{2 k n+k+j}=s_{-2 k+j} \prod_{i=0}^{n} p_{2, j, i}, t_{2 k n+k+j}=t_{-k+j} \prod_{i=0}^{n} q_{2, j, i} . \quad \text { for } n \geq 0, j=1,2,3, \cdots, k .
\end{aligned}
$$

Proof. Since

$$
\begin{gathered}
s_{j}=\alpha s_{-2 k+j}+\frac{A}{t_{-k+j}}=s_{-2 k+j} p_{1, j, 0}, t_{j}=\beta t_{-2 k+j}+\frac{B}{s_{-k+j}}=t_{-2 k+j} q_{1, j, 0}, \\
s_{k+j}=\alpha s_{-k+j}+\frac{A}{t_{j}}=\alpha s_{-k+j}+\frac{A s_{-k+j}}{\beta s_{-k+j} t_{-2 k+j}+B}=s_{-k+j} p_{2, j, 0}, \\
t_{k+j}=\beta t_{-k+j}+\frac{B}{s_{j}}=\beta t_{-k+j}+\frac{B t_{-k+j}}{\alpha t_{-k+j} s_{-2 k+j}+A}=t_{-k+j} q_{2, j, 0} .
\end{gathered}
$$

Hence (6) holds for $n=0, \quad j=1,2,3, \cdots, k$.

For $n=m-1, j=1,2,3, \cdots, k$. assume that (6) is true. $\quad r l_{n}=l_{n+1}-1$,

$$
\prod_{i=0}^{n} p_{1, j, i} \cdot \prod_{i=0}^{n} q_{2, j, i}=r^{n+1}+l_{n+1} \frac{\beta A+B}{t_{-k+j} s_{-2 k+j}}, \quad \prod_{i=0}^{n} q_{1, j, i} \cdot \prod_{i=0}^{n} p_{2, j, i}=r^{n+1}+l_{n+1} \frac{\alpha B+A}{s_{-k+j} t_{-2 k+j}} .
$$

Then for $\#=m, j=1,2,3, \cdots, k$, we have

$$
\begin{aligned}
s_{2 k m+j} & =\alpha s_{2 k(m-1)+j}+\frac{A}{t_{2 k(m-1)+k+j}}=\alpha s_{-2 k+j} \prod_{i=0}^{m-1} p_{1, j, i}+\frac{A}{t_{-k+j} \prod_{i=0}^{m-1} q_{2, j, i}} \\
& =\alpha s_{-2 k+j} \prod_{i=0}^{m-1} p_{1, j, i}+\frac{A}{t_{-k+j}} \cdot \frac{t_{-k+j} s_{-2 k+j} \prod_{i=0}^{m-1} p_{1, j, i}}{r^{m} t_{-k+j} s_{-2 k+j}+l_{m}(\beta A+B)} \\
& =s_{-2 k+j} \prod_{i=0}^{m-1} p_{1, j, i}\left[\alpha+\frac{A}{r^{m} t_{-k+j} s_{-2 k+j}+l_{m}(\beta A+B)}\right]=s_{-2 k+j} \prod_{i=0}^{m} p_{1, j, i}
\end{aligned}
$$




$$
\begin{aligned}
t_{2 k m+j} & =\beta t_{2 k(m-1)+j}+\frac{B}{s_{2 k(m-1)+k+j}}=\beta t_{-2 k+j} \prod_{i=0}^{m-1} q_{1, j, i}+\frac{B}{s_{-k+j} \prod_{i=0}^{m-1} p_{2, j, i}} \\
& =\beta t_{-2 k+j} \prod_{i=0}^{m-1} q_{1, j, i}+\frac{B}{s_{-k+j}} \cdot \frac{s_{-k+j} t_{-2 k+j} \prod_{i=0}^{m-1} q_{1, j, i}}{r^{m} s_{-k+j} t_{-2 k+j}+l_{m}(\alpha B+A)} \\
& =t_{-2 k+j} \prod_{i=0}^{m-1} q_{1, j, i}\left[\beta+\frac{B}{r^{m} s_{-k+j} t_{-2 k+j}+l_{m}(\alpha B+A)}\right]=t_{-2 k+j} \prod_{i=0}^{m} q_{1, j, i} \\
s_{2 k m+k+j} & =\alpha s_{2 k(m-1)+k+j}+\frac{A}{t_{2 k m+j}}=\alpha s_{-k+j} \prod_{i=0}^{m-1} p_{2, j, i}+\frac{A}{t_{-2 k+j} \prod_{i=0}^{m} q_{1, j, i}} \\
& =\alpha s_{-k+j} \prod_{i=0}^{m-1} p_{2, j, i}+\frac{A}{t_{-2 k+j}} \cdot \frac{s_{-k+j} t_{-2 k+j} \prod_{i=0}^{m} p_{2, j, i}}{r^{m+1} s_{-k+j} t_{-2 k+j}+l_{m+1}(\alpha B+A)} \\
& =s_{-k+j} \prod_{i=0}^{m-1} p_{2, j, i}\left[\alpha+\frac{A p_{2, j, m}}{r^{m+1} s_{-k+j} t_{-2 k+j}+l_{m+1}(\alpha B+A)}\right]=s_{-k+j} \prod_{i=0}^{m} p_{2, j, i} \\
t_{2 k m+k+j} & =\beta t_{2 k(m-1)+k+j}+\frac{B}{s_{2 k m+j}}=\beta t_{-k+j} \prod_{i=0}^{m-1} q_{2, j, i}+\frac{B}{s_{-2 k+j} \prod_{i=0}^{m} p_{1, j, i}} \\
& =\beta t_{-k+j} \prod_{i=0}^{m-1} q_{2, j, i}+\frac{B}{s_{-2 k+j}} \cdot \frac{t_{-k+j} s_{-2 k+j} \prod_{i=0}^{m} q_{2, j, i}}{r_{-k+j} s_{-2 k+j}+l_{m+1}(\beta A+B)} \\
& =t_{-k+j} \prod_{i=0}^{m-1} q_{2, j, i}\left[\beta+\frac{B q_{2, j, m}}{r^{m+1} t_{-k+j} s_{-2 k+j}+l_{m+1}(\beta A+B)}\right]=t_{-k+j} \prod_{i=0}^{m} q_{2, j, i}
\end{aligned}
$$

Hence (6) is true.

Theorem 2.2 Let $\left\{\left(x_{n}, y_{n}\right)\right\}_{n=-2 k+1}^{\infty}$ be a positive solution of (1). For $0 \leq j \leq k, n=0,1,2, \cdots$

1) When $r \geq 1$, we have

$$
\begin{gathered}
\lim _{n \rightarrow \infty} x_{n}=\left\{\begin{array}{ll}
0, & \text { when } \alpha>1, \\
+\infty, & \text { when } \alpha<1,
\end{array},\right. \\
\lim _{n \rightarrow \infty} y_{n}= \begin{cases}0, & \text { when } \beta>1, \\
+\infty, & \text { when } \beta<1,\end{cases} \\
\lim _{n \rightarrow \infty} x_{2 k n+j}=a_{-2 k+j}, \\
\lim _{n \rightarrow \infty} y_{2 k n+j}=b_{-2 k+j},
\end{gathered}
$$

where

satisfy:

$$
a_{-2 k+j}, b_{-2 k+j}
$$

$a_{-2 k+j}=\frac{a_{-2 k+j}}{A b_{-k+j} a_{-2 k+j}+\alpha}, b_{-2 k+j}=\frac{b_{-2 k+j}}{B a_{-k+j} b_{-2 k+j}+\beta}$.

2) When $r<1$.

a) Suppose $\frac{\alpha B+A}{\beta A+B}>1$, then $\lim _{n \rightarrow \infty} x_{n}=0, \lim _{n \rightarrow \infty} y_{n}=+\infty$.

b) Suppose $\frac{\alpha B+A}{\beta A+B}<1$, then $\lim _{n \rightarrow \infty} x_{n}=+\infty, \lim _{n \rightarrow \infty} y_{n}=0$.

c) Suppose $\frac{\alpha B+A}{\beta A+B}=1$

i) If $A y_{-k+j} x_{-2 k+j}+\alpha=1$, then $x_{2 k n+j}=x_{-2 k+j}$, for $n=0,1,2, \cdots$.

ii) If $B x_{-k+j} y_{-2 k+j}+\beta=1$, then $y_{2 k n+j}=y_{-2 k+j}$, for $n=0,1,2, \cdots$.

iii) If $A y_{-k+j} x_{-2 k+j}+\alpha \neq 1$, then

$\lim _{n \rightarrow \infty} x_{2 k n+j}=a_{-2 k+j}, \lim _{n \rightarrow \infty} x_{2 k n+k+j}=a_{-k+j}$.

iv) If $B x_{-k+j} y_{-2 k+j}+\beta \neq 1$, then $\lim _{n \rightarrow \infty} y_{2 k n+j}=b_{-2 k+j}, \lim _{n \rightarrow \infty} y_{2 k n+k+j}=b_{-k+j}$. where

satisfy

$$
a_{-2 k+j}, b_{-2 k+j}
$$

$$
a_{-2 k+j}=\frac{a_{-2 k+j}}{A b_{-k+j} a_{-2 k+j}+\alpha}, b_{-2 k+j}=\frac{b_{-2 k+j}}{B a_{-k+j} b_{-2 k+j}+\beta} .
$$

Proof. Note that for $j=1,2, \cdots, k$, 


$$
\begin{gathered}
\lim _{n \rightarrow \infty} p_{1, j, n}=\lim _{n \rightarrow \infty} p_{2, j, n}= \begin{cases}\alpha, & \text { when } r \geq 1 \text { or } \frac{\alpha B+A}{\beta A+B}>1, \\
\frac{\alpha B+A}{\beta A+B}, & \text { when } r<1 \text { or } \frac{\alpha B+A}{\beta A+B}<1,\end{cases} \\
\lim _{n \rightarrow \infty} q_{1, j, n}=\lim _{n \rightarrow \infty} q_{2, j, n}= \begin{cases}\beta, & \text { when } r \geq 1 \text { or } \frac{\beta A+B}{\alpha B+A}>1, \\
\frac{\beta A+B}{\alpha B+A}, & \text { when } r<1 \text { or } \frac{\beta A+B}{\alpha B+A}<1 .\end{cases}
\end{gathered}
$$

Hence

$$
\begin{aligned}
& \lim _{n \rightarrow \infty} \ln \left(s_{2 k n+j}\right)=\ln \left(s_{-2 k+j}\right)+\sum_{n=0}^{\infty} \ln \left(p_{1, j, n}\right)= \begin{cases}+\infty, & \text { when } \alpha>1 \text { or } \frac{\alpha B+A}{\beta A+B}>1, \\
-\infty, & \text { when } \alpha<1 \text { or } \frac{\alpha B+A}{\beta A+B}<1 .\end{cases} \\
& \lim _{n \rightarrow \infty} \ln \left(s_{2 k n+k+j}\right)=\ln \left(s_{-k+j}\right)+\sum_{n=0}^{\infty} \ln \left(p_{2, j, n}\right)= \begin{cases}+\infty, & \text { when } \alpha>1 \text { or } \frac{\alpha B+A}{\beta A+B}>1, \\
-\infty, & \text { when } \alpha<1 \text { or } \frac{\alpha B+A}{\beta A+B}<1 .\end{cases} \\
& \lim _{n \rightarrow \infty} \ln \left(t_{2 k n+j}\right)=\ln \left(t_{-2 k+j}\right)+\sum_{n=0}^{\infty} \ln \left(q_{1, j, n}\right)= \begin{cases}+\infty, & \text { when } \beta>1 \text { or } \frac{\beta A+B}{\alpha B+A}>1, \\
-\infty, & \text { when } \beta<1 \text { or } \frac{\beta A+B}{\alpha B+A}<1 .\end{cases} \\
& \lim _{n \rightarrow \infty} \ln \left(t_{2 k n+k+j}\right)=\ln \left(t_{-k+j}\right)+\sum_{n=0}^{\infty} \ln \left(q_{2, j, n}\right)= \begin{cases}+\infty, & \text { when } \beta>1 \text { or } \frac{\beta A+B}{\alpha B+A}>1, \\
-\infty, & \text { when } \beta<1 \text { or } \frac{\beta A+B}{\alpha B+A}<1 .\end{cases}
\end{aligned}
$$

1) When $r \geq 1$. In view of (4), (6), (7) and (8), we drive

$$
\lim _{n \rightarrow \infty} x_{2 k n+j}=\lim _{n \rightarrow \infty} X_{2 k n+k+j}= \begin{cases}0, & \text { when } \alpha>1, \\ +\infty, & \text { when } \alpha<1,\end{cases}
$$

Thus

$$
\lim _{n \rightarrow \infty} x_{n}= \begin{cases}0, & \text { when } \alpha>1, \\ +\infty, & \text { when } \alpha<1,\end{cases}
$$

Similarly, in view of (4), (6), (9) and (10), we get

$$
\lim _{n \rightarrow \infty} y_{n}= \begin{cases}0, & \text { when } \beta>1, \\ +\infty, & \text { when } \beta<1,\end{cases}
$$

When $\alpha=1$ note that the positive series

$$
\sum_{n=0}^{\infty} \ln \left(p_{1, j, n}\right)=\sum_{n=0}^{\infty} \ln \left(1+\frac{A}{r^{n} t_{-k+j} s_{-2 k+j}+l_{n}(\beta A+B)}\right)
$$

is convergent, in view of (4) and (6), we drive $\lim _{n \rightarrow \infty} x_{2 k n+j}=a_{-2 k+j}$.

Similarly, we get

$$
\lim _{n \rightarrow \infty} x_{2 k n+k+j}=a_{-k+j}, \lim _{n \rightarrow \infty} y_{2 k n+j}=b_{-2 k+j}, \lim _{n \rightarrow \infty} y_{2 k n+j}=b_{-k+j} .
$$

2) When $r<1$.

a) b) The proof is similar to the proof of 1 , we get

$$
\lim _{n \rightarrow \infty} x_{n}=\left\{\begin{array}{ll}
0, & \text { when } \frac{\alpha B+A}{\beta A+B}>1, \\
-\infty, & \text { when } \frac{\alpha B+A}{\beta A+B}<1 .
\end{array},\right.
$$

$$
\lim _{n \rightarrow \infty} y_{n}=\left\{\begin{array}{lc}
0, & \text { when } \frac{\beta A+B}{\alpha B+A}>1, \\
-\infty, & \text { when } \frac{\beta A+B}{\alpha B+A}<1 .
\end{array}\right.
$$

c) Suppose $\frac{\alpha B+A}{\beta A+B}=1$.

i) If $A y_{-k+j} x_{-2 k+j}+\alpha=1$ In view of (1), by induction, we drive $x_{2 k n+j}=x_{-2 k+j}$, for $n=0,1,2, \cdots$.

ii) If $B x_{-k+j} y_{-2 k+j}+\beta=1$, Similarly, we drive 


$$
\begin{aligned}
& y_{2 k n+j}=y_{-2 k+j} \text { for } n=0,1,2, \cdots \\
& p_{1, j, n}=1+\frac{r^{n}\left[(\alpha-1) t_{-k+j} s_{-2 k+j}+A\right]}{r^{n} t_{-k+j} s_{-2 k+j}+l_{n}(\beta A+B)}=1+\frac{r^{n}\left(A y_{-k+j} x_{-2 k+j}+\alpha-1\right)}{r^{n}+y_{-k+j} X_{-2 k+j} l_{n}(\beta A+B)} \\
& q_{1, j, n}=1+\frac{r^{n}\left[(\beta-1) s_{-k+j} t_{-2 k+j}+B\right]}{r^{n} s_{-k+j} t_{-2 k+j}+l_{n}(\alpha B+A)}=1+\frac{r^{n}\left(B x_{-k+j} y_{-2 k+j}+\beta-1\right)}{r^{n}+x_{-k+j} y_{-2 k+j} l_{n}(\alpha B+A)} \\
& q_{2, j, n}=1+\frac{\beta r^{n}\left[(\alpha-1) s_{-k+j} t_{-2 k+j}+A\right]}{r^{n}\left(\beta s_{-k+j} t_{-2 k+j}+B\right)+l_{n}(\beta A+B)}=1+\frac{\beta r^{n}\left(A x_{-k+j} y_{-2 k+j}+\alpha-1\right)}{r^{n}\left(\beta+B x_{-k+j} y_{-2 k+j}\right)+x_{-k+j} y_{-2 k+j} l_{n}(\beta A+B)} \\
& r^{n}\left(\alpha t_{-k+j}\left[(\beta-1) t_{-2 k+j}+A\right)+l_{n}(\alpha B+A)\right.
\end{aligned}
$$

Hence, if $A y_{-k+j} X_{-2 k+j}+\alpha>1$ the positive series

$\sum_{n=0}^{\infty} \ln \left(p_{1, j, n}\right)=\sum_{n=0}^{\infty} \ln \left(1+\frac{r^{n}\left(A y_{-k+j} X_{-2 k+j}+\alpha-1\right)}{r^{n}+y_{-k+j} X_{-2 k+j} l_{n}(\beta A+B)}\right)$

is convergent. If $A y_{-k+j} X_{-2 k+j}+\alpha<1$, the negative series

$$
\sum_{n=0}^{\infty} \ln \left(p_{1, j, n}\right)=\sum_{n=0}^{\infty} \ln \left(1+\frac{r^{n}\left(A y_{-k+j} x_{-2 k+j}+\alpha-1\right)}{r^{n}+y_{-k+j} X_{-2 k+j} l_{n}(\beta A+B)}\right)
$$

is convergent.

In view of (4) and (6), thus if $A y_{-k+j} X_{-2 k+j}+\alpha \neq 1$ then $\lim _{n \rightarrow \infty} x_{2 k n+j}=a_{-2 k+j}$. Similarly, we get

$\lim _{n \rightarrow \infty} x_{2 k n+k+j}=a_{-k+j}$.

iv) If $B x_{-k+j} y_{-2 k+j}+\beta \neq 1$ Similarly, we get $\lim _{n \rightarrow \infty} y_{2 k n+j}=b_{-2 k+j}, \lim _{n \rightarrow \infty} y_{2 k n+j}=b_{-k+j}$.

\section{Acknowledgements}

This work was supported by the NSF of China (No. 11161029), and supported by NSF of Guangxi (No. 2012GXNSFDA276040, 2013GXNSFBA019020), NSF of the Department of Education of Guangxi Province (No. 200103YB157). NSF of Guangxi University of Science and Technology (No. 1166218).

\section{REFERENCES}

[1] A. S. Kurbanlı, C. Ģinar and Ì. Yalçinkaya, "On the Behavior of Positive Solutions of the System of Rational Difference Equation

$$
x_{n+1}=\frac{x_{n-1}}{y_{n} x_{n-1}+1}, y_{n+1}=\frac{y_{n-1}}{x_{n} y_{n-1}+1}, " \text { Mathematical and }
$$

Computer Modelling, Vol. 53, No. 5-6, 2011, pp. 12611267. doi:10.1016/j.mcm.2010.12.009

[2] S. Stević, "On a System of Difference Equations," Appli- ed Mathematics and Computation, Vol. 218, No. 7, 2011, pp. 3372-3378. doi:10.1016/j.amc.2011.08.079

[3] E. Camouzis and G. Papaschinopoulos, "Global Asymptotic Behavior of Positive Solutions on the System of Rational Difference Equations $x_{n+1}=1+\frac{x_{n}}{y_{n-m}}, y_{n+1}=1+\frac{y_{n}}{x_{n-m}}$," Applied Mathematics Letters. Vol. 17, No. 6, 2004, pp. 733-737. doi:10.1016/S0893-9659(04)90113-9

[4] X. Yang, "On the System of Rational Difference Equations $x_{n}=A+\frac{y_{n-1}+a}{x_{n-p} y_{n-p}}, y_{n+1}=A+\frac{x_{n-1}+a}{y_{n-r} x_{n-s}}$, Journal of Mathematical Analysis and Applications, Vol. 307, No. 1, 2005, pp. 305-311. doi:10.1016/j.jmaa.2004.10.045

[5] Q. Wang, F. P. Zeng, G. R. Zhang and X. H. Liu, "Dynamics of the Difference Equation

$x_{n+1}=\frac{\alpha+B_{1} x_{n-1}+B_{3} x_{n-3}+\cdots+B_{2 k+1} x_{n-2 k-1}}{A+B_{0} x_{n}+B_{2} x_{n-2}+\cdots+B_{2 k} x_{n-2 k}}, "$ Journal of

Difference Equations and Applications, Vol. 12, No. 5, 2006, pp. 399-417. doi:10.1080/10236190500453695

[6] Y. Zhang, X. Yang, D. J. Evans and C. Zhu, "On the Nonlinear Difference Equation System

$x_{n+1}=A+\frac{y_{n-m}}{x_{n}}, y_{n+1}=A+\frac{x_{n-m}}{y_{n}}$, , Computers \& Mathematics with Applications, Vol. 53, No. 10, 2007, pp. 15611566. doi:10.1016/j.camwa.2006.04.030

[7] K. S. Berenhaut, J. D. Foley and S. Stević, "The Global Attractivity of the Rational Difference Equation

$y_{n}=\frac{y_{n-k}+y_{n-m}}{1+y_{n-k}+y_{n-m}}, "$ Applied Mathematics Letters, Vol. 20, No. 1,2007 , pp. 54-58. doi:10.1016/j.aml.2006.02.022

[8] K. S. Berenhaut, J. D. Foley and S. Stević, "The Global Attractivity of the Rational Difference Equation $y_{n}=1+\frac{y_{n-k}}{y_{n-m}}$," Proceedings of the American Mathematical Society, Vol. 135, No. 1, 2007, pp. 1133-1140. 
doi:10.1090/S0002-9939-06-08580-7

[9] K. S. Berenhaut, J. D. Foley and S. Stević, "The Global Attractivity of the Rational Difference Equation

$y_{n}=A+\left(\frac{y_{n-k}}{y_{n-m}}\right)^{p}$," Proceedings of the American Mathematical Society, Vol. 136, No. 1, 2008, pp. 103-110. doi:10.1090/S0002-9939-07-08860-0

[10] B. Iričanin and S. Stević, "Eventually Constant Solutions of a Rational Difference Equation," Applied Mathematics and Computation, Vol. 215, No. 2, 2009, pp. 854-856.

$$
\text { doi:10.1016/j.amc.2009.05.044 }
$$

[11] I. Yalcinkaya and C. Ģinar, "Global Asymptotic Stability of Two Nonlinear Difference Equations $z_{n+1}=\frac{t_{n}+z_{n-1}}{t_{n} z_{n-1}+a}$, $t_{n+1}=\frac{z_{n}+t_{n-1}}{z_{n} t_{n-1}+a}$," Fasciculi Mathematici, Vol. 43, 2010, pp. 171-180. 